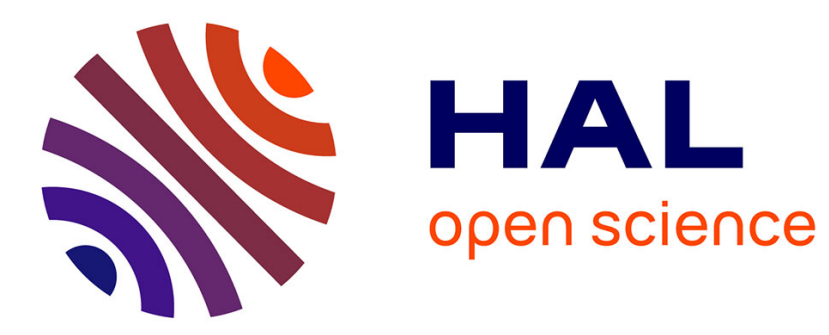

\title{
Lateral forces on a magnet falling in a conducting pipe
} Stéphane Dufour, Gérard Vinsard, J.-P Brancher

\section{To cite this version:}

Stéphane Dufour, Gérard Vinsard, J.-P Brancher. Lateral forces on a magnet falling in a conducting pipe. COMPEL: The International Journal for Computation and Mathematics in Electrical and Electronic Engineering, 2012, 31 (6), pp.1625-1636. 10.1108/03321641211267038 . hal-01498925

\section{HAL Id: hal-01498925 \\ https://hal.univ-lorraine.fr/hal-01498925}

Submitted on 30 Mar 2017

HAL is a multi-disciplinary open access archive for the deposit and dissemination of scientific research documents, whether they are published or not. The documents may come from teaching and research institutions in France or abroad, or from public or private research centers.
L'archive ouverte pluridisciplinaire HAL, est destinée au dépôt et à la diffusion de documents scientifiques de niveau recherche, publiés ou non, émanant des établissements d'enseignement et de recherche français ou étrangers, des laboratoires publics ou privés. 


\title{
Lateral forces on a magnet falling in a conducting pipe
}

\author{
S. Dufour, G. Vinsard and J.-P. Brancher \\ LEMTA, Nancy University, CNRS, 2 avenue de la forêt de Haye, 54516 \\ Vandouvre-lès-Nancy, France
}

\begin{abstract}
Purpose - The purpose of this paper is to investigate the lateral forces during the fall of a magnet in a conducting pipe, when the direction of magnetization of the magnet is fixed. If the direction of magnetization is not parallel to the axis of the pipe, lateral forces occur and a decentration of the magnet happens.

Design/methodology/approach - The problem is studied numerically, with a $\boldsymbol{T}-\boldsymbol{h}$ 3D FE formulation well-suited for the problem. Computational results are compared with experimental results.

Findings - The physical model is given and the main force coefficients analyzed. The lateral forces and the decentration phenomenon are studied as a function of the main parameters (thickness and radius of the pipe).

Originality/value - The direction of magnetization is a key parameter to analyze the dynamics of a magnet motion inside a conducting pipe, when the radii of the pipe and the magnet are not so close. This analysis with a fixed direction of magnetization allows to quantify the lateral forces and the decentration, and is a first step to understand the complete motion which includes the rotation which can be linked to the decentration.

Keywords - Eddy currents, Magnets, Velocity, Finite Element Methods

MSC2010 - 35Q61, 35A10, 35A20, 78M10

Paper type - Research paper
\end{abstract}

\section{Introduction}

The fall of a magnet in a conducting pipe is a classical academic illustration of forces generated by eddy currents (Levin et al. 2006, Partovi et al. 2006, Roy et al. 2007). The corresponding engineering application is the building of magnetic dampers (Bae et al., 2009). In these cases, the radii of the magnet and of the pipe are close.

Analytical studies of the steady-state solution have been made over the years. All of them consider that the magnet falls along the axis of symmetry with a direction of magnetization of the magnet parallel to its axis, since analytical solutions can be found due to the axisymmetry. Then the different parameters are analysed, such as the 
conductivity (Iniguez et al. 2004, Levin et al. 2006), the pipe thickness (Donoso et al. 2009), and the excentricity with the assumption of an off-axis longitudinal motion (Donoso et al. 2011).

However when the radius of the pipe is slightly larger than the one of the magnet, it has been noticed by experiment, that the position of the magnet (and the direction of magnetization) oscillates around the axis of symmetry. After having checked many reasons, an explanation is that a dynamical equilibrium state occurs either when the starting direction of magnetization is not parallel to the axis of symmetry, or when the starting position is not on the axis of symmetry.

When the radius of the pipe is even larger, then the magnet rotates around itself at an almost constant speed and it oscillates around a mean position which is not the axis of symmetry. The fall of a magnet near a plane represents in fact the limit case of a pipe of infinite radius (Davis 1972).

A full dynamical model of the motion of the magnet (valid in all these cases) requires to consider five degrees of freedom (dof): six for a solid minus the one of the rotation of the magnet around its axis of symmetry, that is the three dof of the position of the center and the two dof of the direction of magnetization. It requires a huge amount of work to build the expression of the system. The generalized force, which corresponds to the orientation of each of these dof, has to be computed, and the crossed effects have to be taken into account.

A step in such a work is to fix the direction of magnetization (which is the case of magnetic dampers), and to investigate only the forces along the axis of symmetry (brake forces) and the ones perpendicular to this axis (lateral forces): this step is the aim of the paper. Moreover, the validity of the computation will be controlled by experiment.

As the motion of the magnet mainly occurs in the plane described by the axis of symmetry and the starting position of the center of the magnet, the direction of magnetization is only considered in this plane. Lateral forces occur when the direction of magnetization is neither parallel nor perpendicular to the axis (even if the magnet is centered on the axis of the pipe).

This can be understood qualitatively in terms of equivalent wires where the magnet is considered as the superposition of two coils, one with its normal directed along the axis and the other with orthogonal normal. In the motion along the axis the normal directed coil induces circular loop currents with a normal also directed along the axis. The value of the mutual inductance between these last currents (considered as coils) and the coil directed orthogonally depends on the position of the magnet with respect to the axis; and in particular its derivative, which varies as the lateral force exerted on the magnet when the currents are fixed, is not zero on the axis.

Computing the forces may be done by different ways, analytically or numerically. On the one hand, when the direction of magnetization is perpendicular to the axis of the pipe, full analytical solutions are quite elaborate to find because of the complex paths of the current lines (Fig. 3, current lines obtained numerically). 
On the other hand, a finite element classical magnetodynamic model may be used. However, it is a bit difficult to handle the computations at any scale of magnet and pipe, to take into account the lateral motion, and the different directions of magnetization, as they are linked to the limitations of the FE package that is used (in particular the mesh generator).

Thus, a mixed approach, with suitable 3D finite element formulations, valid for low induced magnetic fields, has been searched, in order to consider only the pipe. These formulations have been used with Taylor expansions, to find the dependencies of the brake and lateral forces as functions of the speeds. It allows to find the coefficients of the Taylor expansion with successive computations, like with analytical methods.

The different parameters of the problem are for the pipe, its inner radius, thickness and conductivity, and for the magnet its radius and magnetization intensity. The dependence of the forces with respect to the conductivity and magnetization intensity is linear. The radius of the magnet will be fixed, and the pipe's inner radius and thickness will be the effective studied parameters, to consider the lateral and brake forces of a magnet falling in a pipe as a function of the decentration and direction of magnetization.

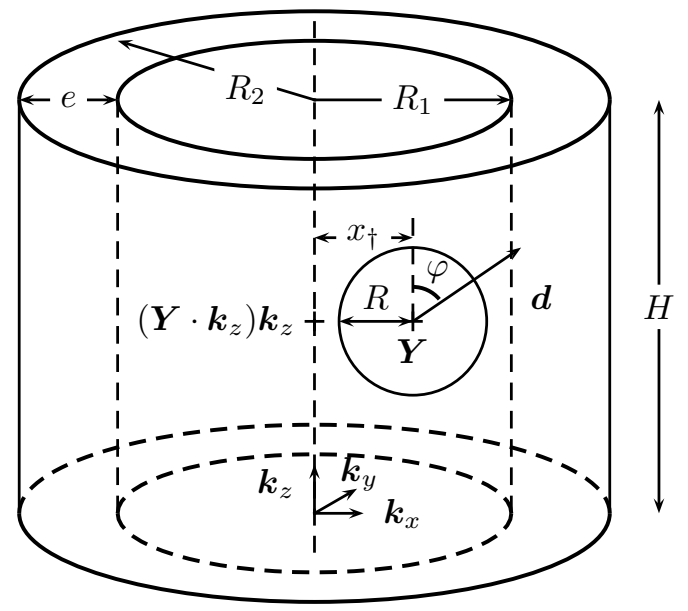

Figure 1.

\section{Position of the Problem}

The axis of the pipe (interior and exterior radii $R_{1}$ and $R_{2}$ ) is along the $z$-direction (Figure 1). The position of the center of the spherical magnet (radius $R$ ) is in a fixed frame $\boldsymbol{Y}(t)$ and its direction of magnetization $\boldsymbol{d}$.

The motion of the magnet generates magnetic flux variations and then electromotive forces that drive eddy currents in the pipe. For completeness it would be necessary to take into account both the longitudinal and lateral motions as source of eddy cur- 
rents; and then it would be impossible to find an expression of the force that only depends on the state variables of the motion.

Fortunately, the essential part of the motion is the longitudinal motion with a speed almost constant; then the lateral motion or the fluctuations around this constant speed are low and the corresponding eddy currents are negligible.

The source field of a spherical magnet (Jackson 1999) is in this fixed frame:

$$
\boldsymbol{h}_{s}=\frac{M R^{3}}{|\boldsymbol{y}-\boldsymbol{Y}(t)|^{3}}\left(\frac{\boldsymbol{d} \cdot(\boldsymbol{y}-\boldsymbol{Y}(t))}{|\boldsymbol{y}-\boldsymbol{Y}(t)|^{2}}(\boldsymbol{y}-\boldsymbol{Y}(t))-\frac{\boldsymbol{d}}{3}\right)
$$

where $\boldsymbol{y}$ and $\boldsymbol{Y}$ are respectively the considered position and the position of the center of the magnet, $M$ is the magnetization intensity and $\boldsymbol{d}$ its direction in space. The position of the center of the magnet is and its speed are:

$$
\boldsymbol{Y}=x_{\dagger} \boldsymbol{k}_{x}+z \boldsymbol{k}_{z} \quad \text { and } \quad \boldsymbol{v}=\dot{x}_{\dagger} \boldsymbol{k}_{x}+\dot{z} \boldsymbol{k}_{z}
$$

As the speed in the vertical direction is almost constant, it is interesting to consider a moving frame by displacing vertically the center of the fixed frame at speed of

$$
\dot{\boldsymbol{Y}}(t) \cdot \boldsymbol{k}_{z}=-\dot{z} \boldsymbol{k}_{z}
$$

In this moving frame the current point $x$ is linked to $y$ by:

$$
\boldsymbol{x}=\boldsymbol{y}-\left(\boldsymbol{Y} \cdot \boldsymbol{k}_{z}\right) \boldsymbol{k}_{z}
$$

and then the position of the magnet is $\boldsymbol{X}=x_{\dagger} \boldsymbol{k}_{x}$. The magnetic source field of the magnet is (outside the magnet):

$$
\boldsymbol{h}_{s}=\frac{M R^{3}}{|\boldsymbol{x}-\boldsymbol{X}(t)|^{3}}\left(\frac{\boldsymbol{d} \cdot(\boldsymbol{x}-\boldsymbol{X}(t))}{|\boldsymbol{x}-\boldsymbol{X}(t)|^{2}}(\boldsymbol{x}-\boldsymbol{X}(t))-\frac{\boldsymbol{d}}{3}\right)
$$

$\boldsymbol{d}$ is assumed to be in the $(x, z)$ plane as the motion itself:

$$
\boldsymbol{d}=\sin \varphi \boldsymbol{k}_{x}+\cos \varphi \boldsymbol{k}_{z}
$$

The source electric field (EMF) corresponding to the magnet motion is

$$
\boldsymbol{e}_{s}=\mu_{0} \boldsymbol{v} \times \boldsymbol{h}_{s}=\mu_{0}\left(\dot{x}_{\dagger} \boldsymbol{k}_{x}+\dot{z} \boldsymbol{k}_{z}\right) \times \boldsymbol{h}_{s}
$$

where $\boldsymbol{h}$ is the magnetic field generated by the eddy currents density $\boldsymbol{j}$, that is:

$$
\nabla \times h=j \quad ; \quad \nabla \cdot h=0
$$

the current density verifies:

$$
\nabla \cdot j=0
$$


then it is linked to the EMF by the Ohm law:

$$
\boldsymbol{j}=\sigma\left(\boldsymbol{e}_{s}+\boldsymbol{e}_{i}+\boldsymbol{\nabla} \phi\right)
$$

where $\sigma$ is the conductivity, $\phi$ the electrical scalar potential (needed to insure the free-divergence of the induced current density) and $e_{i}$ the EMF corresponding to the induced current motion:

$$
\boldsymbol{e}_{i}=\mu_{0} \dot{z} \boldsymbol{k}_{z} \times \boldsymbol{h}
$$

There is no term in $\mu_{0} \dot{x}_{\dagger} \boldsymbol{k}_{x} \times \boldsymbol{h}_{s}$ in (11) since the induced current only moves in the $z$-direction. The rotational of (10) in the pipe leads to:

$$
\boldsymbol{\nabla} \times \frac{\boldsymbol{j}}{\sigma}=\mu_{0} \boldsymbol{\nabla} \times\left(\boldsymbol{v} \times \boldsymbol{h}_{s}\right)+\mu_{0} \boldsymbol{\nabla} \times\left(\dot{z} \boldsymbol{k}_{z} \times \boldsymbol{h}\right)
$$

And finally, the force exerted on the magnet is the opposite of the (Laplace) force exerted on the pipe:

$$
\boldsymbol{f}=-\mu_{0} \int_{D} \boldsymbol{j} \times \boldsymbol{h}_{s} d \boldsymbol{x}^{3}
$$

where the induced magnetic field is not considered because the induced currents have no global effects on themselves. The steady-state assumption allows to express this force as a function of the state variables $x_{\dagger}, \dot{x}_{\dagger}, z, \dot{z}$ of the magnet (in addition to the geometrical parameters $R_{1}$ and $R_{2}$ of the pipe and of its electrical conductivity). And the next section explains the used method to obtain this result through numerical computations.

\section{Numerical Model}

We use the current function $\boldsymbol{T}$ in the conducting region $D$ such as:

$$
j=\nabla \times T
$$

(to satisfy the free-divergence of $\boldsymbol{j}$ in $D$ ), submitted to the conditions

$$
\begin{array}{lll}
\boldsymbol{\nabla} \cdot \boldsymbol{T}=0 & \text { in } & D \\
\boldsymbol{T} \times \boldsymbol{n}=\mathbf{0} & \text { on } & \partial D
\end{array}
$$

which insure $\boldsymbol{j} \cdot \boldsymbol{n}=0$ on $\partial D$ and the uniqueness of $\boldsymbol{T}$.

The magnetic vector potential (MVP) is computed by using the Biot-Savart law:

$$
\boldsymbol{a}(\boldsymbol{x})=\frac{\mu_{0}}{4 \pi} \int_{D} \frac{\boldsymbol{\nabla} \times \boldsymbol{T}(\boldsymbol{y})}{|\boldsymbol{x}-\boldsymbol{y}|} d \boldsymbol{y}^{3}
$$

The induced magnetic field $\boldsymbol{h}$ solution of (8) is then obtained with:

$$
\boldsymbol{h}=1 / \mu_{0} \boldsymbol{\nabla} \times \boldsymbol{a}
$$




\subsection{Electromagnetic fields}

In a certain sense, the use of both current function $\boldsymbol{T}$ and magnetic field $\boldsymbol{h}$ is a mixed formulation (Dular et al., 1997). It is in fact possible to compute the values of these variables in one step (strong coupling), with classical $\boldsymbol{T}-\Omega$ or $\boldsymbol{a}-\phi$ methods. The main advantage to consider separately the computation of $\boldsymbol{T}$ and $\boldsymbol{h}$ (Dufour et al., 2007 ) is that only the conducting domain can be considered, whereas other methods need to mesh the domains outside the conducting domain, in particular the magnet (which raises numerical difficulties to get the appropriate forces when the magnet has a lateral motion).

Let us consider the solution $\boldsymbol{U}$, which depends on a given vector field $\boldsymbol{W}$, of the generic variational problem: $\forall \boldsymbol{T}^{\prime}$ verifying (15)

$$
\int_{D}\left(\boldsymbol{\nabla} \times \boldsymbol{U}-\sigma \mu_{0} \boldsymbol{W}\right) \cdot \boldsymbol{\nabla} \times \boldsymbol{T}^{\prime} d \boldsymbol{x}^{3}=0
$$

under the constraint:

$$
\boldsymbol{\nabla} \cdot \boldsymbol{U}=0 \text { in } D
$$

This dependence of $\boldsymbol{U}$ with respect to $\boldsymbol{W}$ is summarized by the introduction of a shorthand notation (functional operator) $\mathcal{G}$ that associates the solution $\boldsymbol{U}$ of (18-19) to the considered vector field $\boldsymbol{W}$ :

$$
\boldsymbol{U}=\mathcal{G}(\boldsymbol{W})
$$

Then the current function $\boldsymbol{T}$ defined by (14) with (15) and (12) is

$$
\boldsymbol{T}=\mathcal{G}\left(\boldsymbol{v} \times \boldsymbol{h}_{s}+\dot{z} \boldsymbol{k}_{z} \times \boldsymbol{h}\right)
$$

Nodal finite elements with a penalty method (Ribaki et al., 1990) to enforce the freedivergence (19) are used to compute $\boldsymbol{T}$. The MVP $\boldsymbol{a}$ is a function of the current function $\boldsymbol{T}$ with the help of the Biot-Savart law (16). Then $\boldsymbol{a}$ is computed on the nodes of the tetrahedral mesh of $D$. The Gauss quadrature rule may be used but has the drawback that the integral contains singularities for the tetrahedron belonging to the node where $\boldsymbol{a}$ is computed. A treatment with locally-corrected quadrature rules for these tetrahedrons may be done (Strain, 1995) but we have preferred to separate and treat analytically (with formulae issued from a symbolic computation) the singular parts of the integrals to keep simultaneously a low order of Gauss quadrature and enough accuracy.

The next step is to compute the induced magnetic field: it is obtained as piecewise on the element vector field by the direct differentiation (17) of the linear approximation of the MVP in the element obtained by the Biot-Savart law (16). These operations are summarized once again by the introduction of the shorthand notation:

$$
\boldsymbol{h}(\boldsymbol{x})=\mathcal{F}(\boldsymbol{T}(\boldsymbol{x}))=\frac{1}{4 \pi} \nabla_{[\boldsymbol{x}]} \times \int_{D} \frac{\nabla_{[\boldsymbol{y}]} \times \boldsymbol{T}(\boldsymbol{y})}{|\boldsymbol{x}-\boldsymbol{y}|} d \boldsymbol{y}^{3}
$$


The processes where $\boldsymbol{T}$ is computed for a given $\boldsymbol{h}$ with (21) (electric step) and $\boldsymbol{h}$ for a given $\boldsymbol{T}$ with (22) (magnetic step) may not converge. Such lack of convergence occurs if the magnetic field $\boldsymbol{h}$ play a major role in the EMF. Fortunately in the case where the speed of fall corresponds to a low magnetic Reynolds number $\sigma \mu_{0} R_{1}|\dot{z}|$ (less than unity for speeds below $2 \mathrm{~m} / \mathrm{s}$ ), the convergence happens and moreover is quick.

From a practical point of view, the iterative finding of $(\boldsymbol{T}, \boldsymbol{h})$ which is done by (21)-(22) is eased by a Taylor expansion of the vectors fields as a function of the speeds $\left(\dot{x}_{\dagger}, \dot{z}\right)$ :

$$
\begin{aligned}
\boldsymbol{T} & =\sum_{n=0}^{\infty} \dot{z}^{n}\left(\boldsymbol{T}_{n x} \dot{x}_{\dagger}+\boldsymbol{T}_{n z} \dot{z}\right) \\
\boldsymbol{h} & =\sum_{n=0}^{\infty} \dot{z}^{n}\left(\boldsymbol{h}_{n x} \dot{x}_{\dagger}+\boldsymbol{h}_{n z} \dot{z}\right)
\end{aligned}
$$

Then the zero-order fields verify:

$$
\begin{array}{llll}
\boldsymbol{T}_{0 x}=\mathcal{G}\left(\boldsymbol{k}_{x} \times \boldsymbol{h}_{s}\right) & \text { and } & \boldsymbol{h}_{0 x}=\mathcal{F}\left(\boldsymbol{T}_{0 x}\right) \\
\boldsymbol{T}_{0 z}=\mathcal{G}\left(\boldsymbol{k}_{z} \times \boldsymbol{h}_{s}\right) & \text { and } & \boldsymbol{h}_{0 z}=\mathcal{F}\left(\boldsymbol{T}_{0 z}\right)
\end{array}
$$

They correspond to the case where the induced EMF is neglected. The next order terms verify: for $j \in\{x, z\}$ and $n \geq 0$

$$
\boldsymbol{T}_{(n+1) j}=\mathcal{G}\left(\boldsymbol{k}_{z} \times \boldsymbol{h}_{n j}\right) \quad \text { and } \quad \boldsymbol{h}_{(n+1) j}=\mathcal{F}\left(\boldsymbol{T}_{(n+1) j}\right)
$$

\subsection{Force components}

The iterative computation of $\boldsymbol{T}$ allows to find directly the force as a function of the speeds, since the force (obtained with (13)) is linear with respect to $\boldsymbol{T}$ and then is (formally) a series as:

$$
\boldsymbol{f}=\sum_{n=0}^{\infty} \dot{z}^{n}\left(\boldsymbol{f}_{n x} \dot{x}_{\dagger}+\boldsymbol{f}_{n z} \dot{z}\right)
$$

where the coefficients depend on $\varphi$ and $x$. The components of the Laplace force (26) are such as: for $(i, j) \in\{x, z\}^{2}$ and $n \geq 0$

$$
\boldsymbol{f}_{n i} \cdot \boldsymbol{k}_{j}=\int_{D} \frac{1}{\sigma} \boldsymbol{\nabla} \times \boldsymbol{T}_{0 j} \cdot \boldsymbol{\nabla} \times \boldsymbol{T}_{n i} d \boldsymbol{x}^{3}
$$

For $n \geq 1$, these components can also be written as:

$$
\boldsymbol{f}_{n i} \cdot \boldsymbol{k}_{j}=\mu_{0} \int_{D}\left(\boldsymbol{h}_{(n-1) i} \times\left(\boldsymbol{\nabla} \times \boldsymbol{T}_{0 j}\right)\right) \cdot \boldsymbol{k}_{j} d \boldsymbol{x}^{3}
$$

The advantage of (28) with respect to (27) is that only the $(n-1)$-order field components are needed for the computation of the $n$-order force components. 
The Taylor expansion of the force as a function of the speeds is well suited to analyse the dependencies of each coefficient on $\varphi$ and $x_{\dagger}$. Since the lateral $\dot{x}_{\dagger}$ and longitudinal $\dot{z}$ speeds are low, (26) is limited to the second order only as a function of $\dot{z}$ and $\dot{x}_{\dagger}$ : higher orders have been investigated and are negligible. The first order components are computed with (27) and the second order with (28) for $n=1$ : only the computation of the zero-order fields $\left(\boldsymbol{T}_{0 x}, \boldsymbol{h}_{0 x}, \boldsymbol{T}_{0 z}, \boldsymbol{h}_{0 z}\right)$ is necessary.

As the source field (5) is a sinusoidal function of $\varphi$ these components depend sinusoidally on $2 \varphi$. The dependence on $x_{\dagger}$ is more complicated, however as our objective is to determine the tendencies of the motion near the centered position $x_{\dagger}=$ 0 , we bound the dependence to the first order on $x_{\dagger}$. We obtain then

$$
\begin{aligned}
& F_{x} \simeq-\dot{x}_{\dagger} \alpha_{x}\left(1-\beta_{x} \cos (2 \varphi)\right)+\gamma \sin (2 \varphi) \dot{z}-\kappa x_{\dagger} \dot{z}^{2} \\
& F_{z} \simeq-\dot{z} \alpha_{z}\left(1+\beta_{z} \cos (2 \varphi)\right)+\gamma \sin (2 \varphi) \dot{x}_{\dagger}-\kappa x_{\dagger} \dot{x}_{\dagger} \dot{z}
\end{aligned}
$$

where $F_{x}=\boldsymbol{f} \cdot \boldsymbol{k}_{x}$ and $F_{z}=\boldsymbol{f} \cdot \boldsymbol{k}_{z}$ are the lateral and axial forces and where the constants $\left(\alpha_{x}, \beta_{x}, \alpha_{z}, \beta_{z}, \gamma, \kappa\right)$ can be computed by with $\left(\boldsymbol{T}_{0 i}, \boldsymbol{h}_{0 j}\right)$.

The terms $-\alpha_{z}\left(1+\beta_{z} \cos (2 \varphi)\right)$ and $-\alpha_{x}\left(1-\beta_{x} \cos (2 \varphi)\right)$ are exactly $\boldsymbol{f}_{0 z} \cdot \boldsymbol{k}_{z}$ and $\boldsymbol{f}_{0 x} \cdot \boldsymbol{k}_{x}$, computed with (27); the $\alpha_{z / x}, \beta_{z / x}$ coefficients are identified by using two computations with two directions of magnetization: one in the fall $(\varphi=0)$ and the other in the lateral $(\varphi=\pi / 2)$ directions. We obtain $0<\beta_{z / x}<1$ (in accordance to the fact that the fall speed is lower for $\varphi=0$ than $\varphi=\pi / 2$ for the $z$ coefficient). Since $\left.\partial_{x} \boldsymbol{h}_{s}\right|_{\varphi=0}=\left.\partial_{z} \boldsymbol{h}_{s}\right|_{\varphi=\pi / 2}$, the fields $\left.\boldsymbol{T}_{n x}\right|_{\varphi=0}$ and $\left.\boldsymbol{T}_{n z}\right|_{\varphi=\pi / 2}$ are the same. It leads to the relation $\alpha_{z}\left(1-\beta_{z}\right)=\alpha_{x}\left(1-\beta_{x}\right)$.

The term $\gamma \sin (2 \varphi)$ is $\boldsymbol{f}_{0 z} \cdot \boldsymbol{k}_{x}$ or equivalently $\boldsymbol{f}_{0 x} \cdot \boldsymbol{k}_{z}$ due to the symmetry of (27); it is the source term of the decentration. It happens when the set direction of magnetization is neither parallel nor perpendicular to the axis of the tube, even if the magnet is centered in the pipe. To explain the presence of this term, we can consider that the main component of $\boldsymbol{T}_{0 x}$ is its radial component $T_{0 x r}$, then $\gamma \simeq$ $\left.\left.\mu_{0} \int_{D} \partial_{z} T_{0 x r}\right|_{\frac{\pi}{4}} h_{s r}\right|_{\frac{\pi}{4}} d \boldsymbol{x}^{3}\left(h_{s r}\right.$ being the radial component of $\left.\boldsymbol{h}_{s}\right)$ can be computed by:

$$
\gamma \simeq \mu_{0} \int_{D}\left(\left.\left.\partial_{z} T_{0 x r}\right|_{\frac{\pi}{2}} h_{s r}\right|_{0}+\left.\left.\partial_{z} T_{0 x r}\right|_{0} h_{s r}\right|_{\frac{\pi}{2}}\right) d \boldsymbol{x}^{3}
$$

Figure 2 shows the different fields of (31) with their directions (in the inner radius of the pipe $\left.\left|\boldsymbol{x} \cdot \boldsymbol{k}_{r}\right|=R_{1}\right)$. Due to the symmetries of the fields $\left(\left.h_{s r}\right|_{\varphi=0}\right.$ and $\left.T_{0 x r}\right|_{\varphi=\pi / 2}$ are symmetric with respect to the plane $z=0$, whereas $\left.h_{s r}\right|_{\varphi=\pi / 2}$ and $\left.T_{0 x r}\right|_{\varphi=0}$ are anti-symmetric), $\left.\boldsymbol{f}_{0 x}\right|_{\varphi=0} \cdot \boldsymbol{k}_{z}$, which is approximately equal to $\left.\left.\int_{D} \partial_{z} T_{0 x r}\right|_{0} h_{s r}\right|_{0} d \boldsymbol{x}^{3}$, vanishes. For the same reasons $\left.\boldsymbol{f}_{0 x}\right|_{\varphi=\pi / 2} \cdot \boldsymbol{k}_{z}$ is also null. It is not the case for the term $\partial_{z} T_{0 x r}\left|\frac{\pi}{2} h_{s r}\right|_{0}$ : it has two vortices, centered on $z=0$. The term $\left.\left.\partial_{z} T_{0 x r}\right|_{\frac{\pi}{2}} h_{s r}\right|_{0}$ has four vortices in the opposite direction, thus the integral of their sum $\gamma$ is not zero.

And last, the term $-\kappa x_{\dagger} \dot{z}^{2}$ is $\boldsymbol{f}_{1 z} \cdot \boldsymbol{k}_{x}$; On the contrary to the previous coefficients which can be found with $\boldsymbol{T}_{0(x / z)}$ and (27), the computation of $\kappa$ needs $\boldsymbol{h}_{0(x / z)}$ and (28). The term $-\kappa x_{\dagger} \dot{z}^{2}$ is analogous to a restoring force that tends to center the magnet on the axis. 

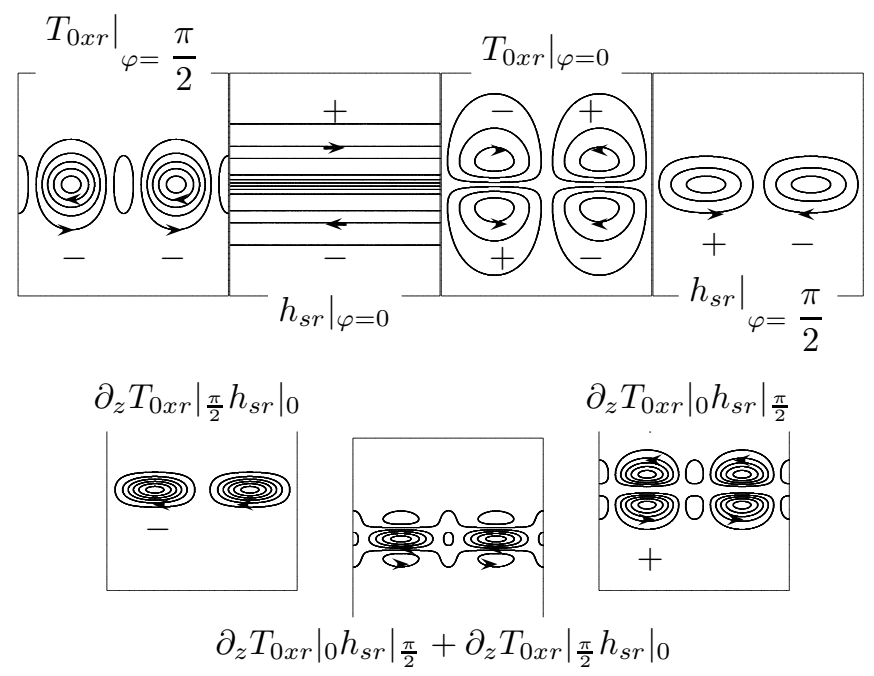

Figure 2.

Fields used for the computation of the decentration term

\section{Results}

A spherical Nd-Fe-B magnet (of radius $R=6.35 \mathrm{~mm}$, mass $m=8 \mathrm{~g}$, magnetization $\mu_{0} M=0.7 \mathrm{~T}$ ) and copper pipe (of radius $R_{1}=8 \mathrm{~mm}$, length $H=1 \mathrm{~m}$, conductivity $\left.\sigma=510^{7} \mathrm{~S} / \mathrm{m}\right)$ is considered.

The accuracy of the $\boldsymbol{T}-\boldsymbol{h}$ formulation has been verified by a comparison with a classical $\boldsymbol{T}-\Omega$ formulation: the agreement is good. The power loss has been computed in both cases (with the same mesh for $D$ ) for several thickness $(1 \mathrm{~mm}$ $\leq e \leq 8 \mathrm{~mm}$ ): the difference is less than $0.5 \%$.

\subsection{Centered Magnet}

The magnet is supposed to be centered in this section, and the investigated parameters are the thickness and the inner radius of the pipe ( $\left.e=0.5-8 \mathrm{~mm}, R_{1}=8-16 \mathrm{~mm}\right)$.

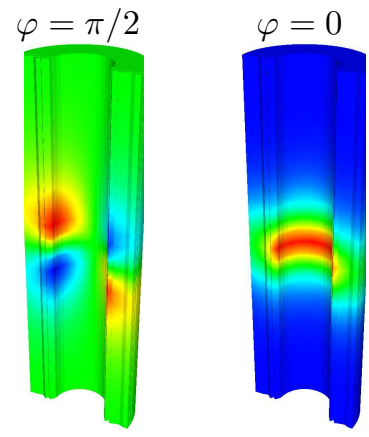

$e=8 \mathrm{~mm}$

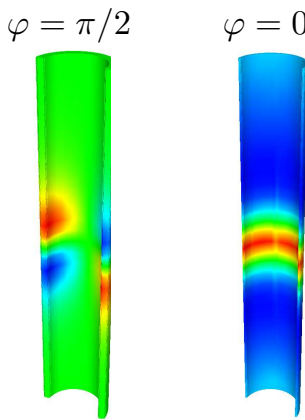

$e=0.5 \mathrm{~mm}$
Figure 3.

Lines fields of $\boldsymbol{T}_{0 z} \cdot \boldsymbol{k}_{r}$ (main component of the current function) 
Figure 3 shows the current lines of $T_{0 z r}$, the main component (along the radial direction) of $\boldsymbol{T}_{0 z}$ for two directions of magnetization on one half of the tube. For a small thickness $(e=0.5 \mathrm{~mm}), T_{0 z r}$ is almost constant as a function of $r$. If the direction of magnetization is in the fall direction $(\varphi=0)$, the field lines are axisymmetric. For $\varphi=\pi / 2$, the cut plane is parallel to the direction of magnetization. $T_{0 z r}$ is an odd function of $z$ and even function of $y$. For a large thickness $(e=8 \mathrm{~mm}), T_{0 z r}$ decreases as a function of $r$, and it is almost negligible for $r>3 \mathrm{~mm}$. The thickness of the pipe is an important factor: the problem is three-dimensional for large thickness, whereas a model reduction in the $r$ direction may be acceptable for lower thickness. In this case a shell current approximation may be used, where the induced current is assumed to be concentrated in a shell and the thickness is taken into account in an average treatment of (12).
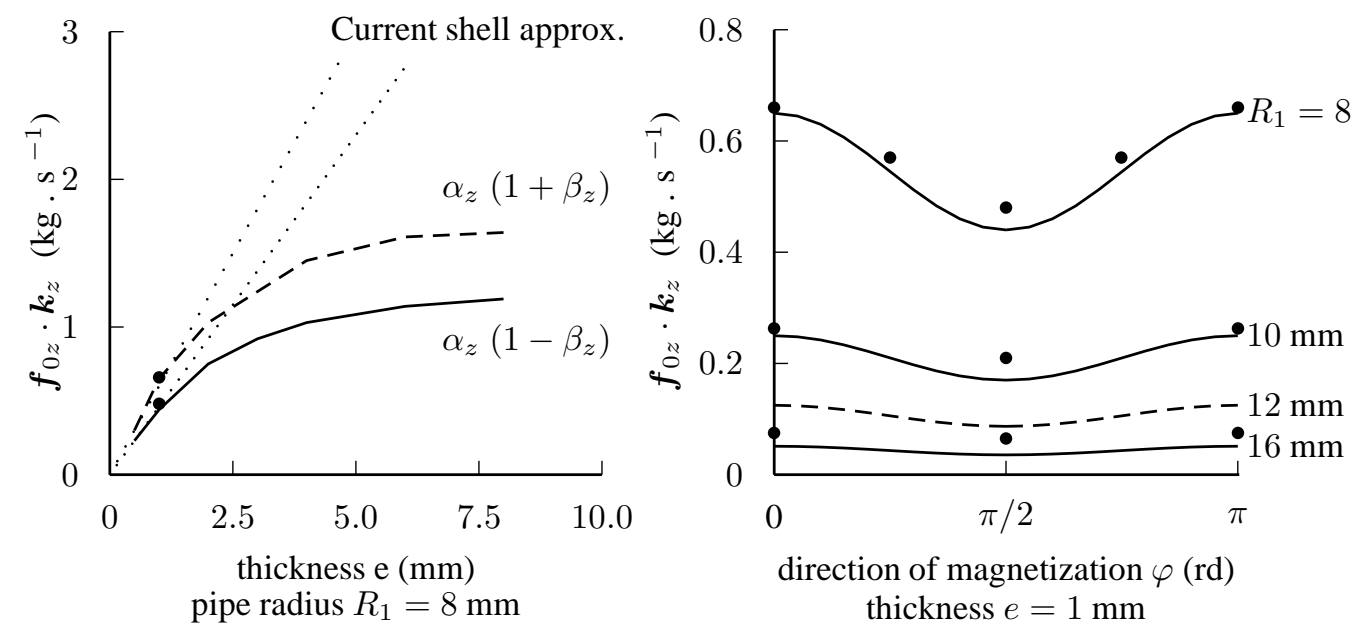

Figure 4. Brake force coefficients $\boldsymbol{f}_{0 z} \cdot \boldsymbol{k}_{z}$ as a function of the pipe thickness (left) and the direction of magnetization (right) without decentration

Figure 4 (left) shows the brake force coefficients of (30) $\alpha_{z}\left(1+\beta_{z} \cos (2 \varphi)\right)$, for $\varphi=0$ and $\varphi=\pi / 2$, versus the thickness of the pipe, for a constant inner radius of the pipe. For small values, the coefficients are almost linear with respect to the thickness: the $r$-dependence of the currents is low. In this case the shell current approximation (small dots) may be used: it valid for a thickness smaller than $2 \mathrm{~mm}$.

Above a thickness of $6 \mathrm{~mm}$ the brake force coefficients are almost constant: the induced currents vanish at the outer radius for these depths.

The brake force coefficients of (30) are investigated as a function of the direction of magnetization for several pipe radii $R_{1}$ (Fig. 4 (right)). The variation of the brake force between maximum and minimum as a function of the direction of magnetization is about one third.

The computation has also been compared to experiment. The measurement of the brake force for a given direction of magnetization has been investigated. The problem is to insure a fixed direction of magnetization. Instead of a fall, a system of rope 

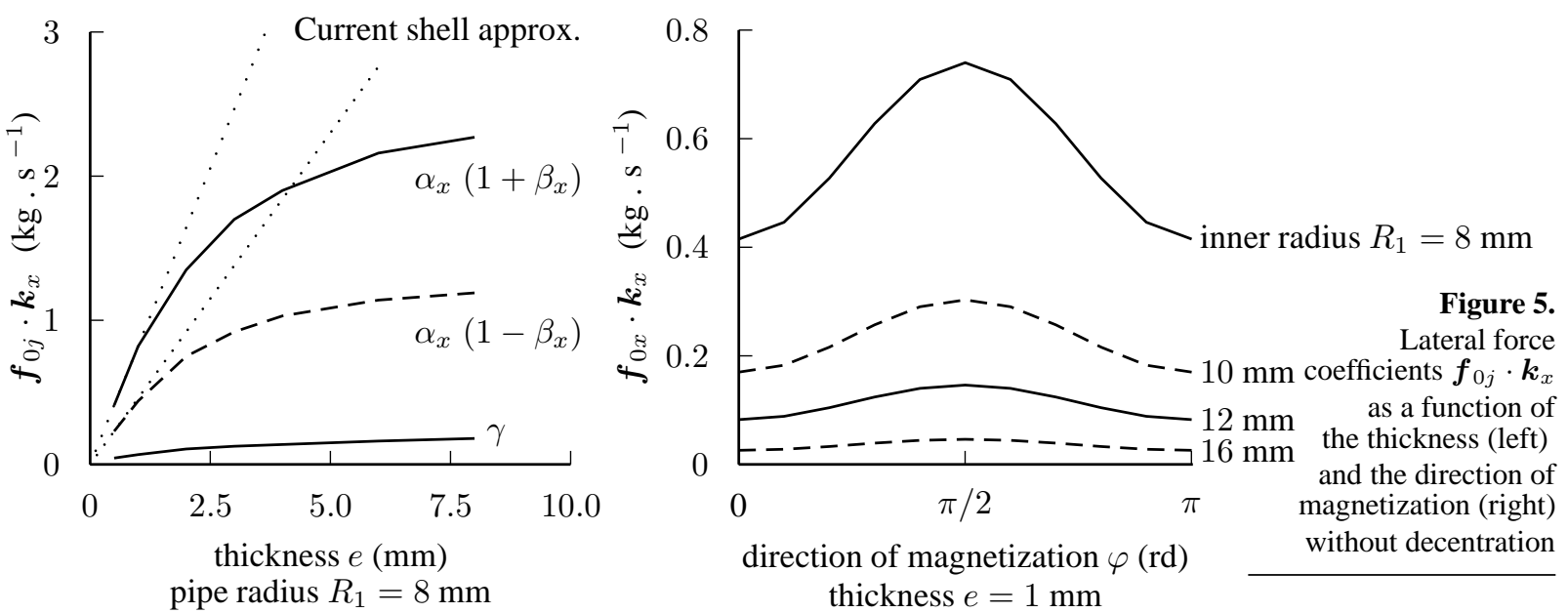

pulley and counterweight has been built to lift the magnet, and control the speed. The frictional force of the pulley has been measured with the help of a non-magnetized sphere. It is then possible to get the brake force as a function of the direction of magnetization. These experimental values are displayed with the larger dots. The model predicts quite well the variation of the brake force as a function of the direction of magnetization for different inner radii of the pipe $(8-10-16 \mathrm{~mm})$.

Figure 5 (left) shows the lateral force coefficients of (29) $\alpha_{x}\left(1-\beta_{x} \cos (2 \varphi)\right)$, for $\varphi=0$ and $\varphi=\pi / 2$. The same current shell approximation can be made for values of thickness lower than $2 \mathrm{~mm}$. If the lateral speed is of the same order of magnitude as the vertical speed, then the main term of the lateral force is in this case $\boldsymbol{f}_{0 x} \cdot \boldsymbol{k}_{x}$ : it is of the same order of magnitude as the brake force (Fig. 5 (right)). The decentration force (term in $\gamma$ ) is about one tenth of the brake force for $\varphi=\pi / 4$, which corresponds to its maximum value.

\subsection{Decentration of the magnet}

For a constant decentration, the lateral forces are the decentration term $\gamma \sin (2 \varphi) \dot{z}$ balanced by the restoring force $-\kappa \dot{z}^{2} x_{\dagger}$.

This first-order approximation of the restoring force on $x_{\dagger}$ was made in section 3.2 to obtain a simple expression of the restoring force near the axis: it comes from the Taylor expansion of $\boldsymbol{h}_{s}(5)$ to the first order as a function of $x_{\dagger}$. The computations can also be made by using an effective decentration $x_{\dagger}$ for $\boldsymbol{h}_{s}$ (i.e. by entering the effective lateral position $\boldsymbol{X}$ in (5)).

The comparison between the first-order approximation and the less approximated model is shown (Fig. 5) for some values of internal radius of the pipe. The first-order approximation is valid for low decentrations (in particular for the pipe with $R_{1}=8$ $\mathrm{mm})$. For higher decentrations, the restoring force becomes a cubic function, and a 
third-order approximation could be more accurate.

The linear approximation gives as computed lateral equilibrium position $x_{\dagger}=8$ $\mathrm{mm}$ for $R_{1}=12 \mathrm{~mm}$ (beyond the validity of the first-order approximation of the restoring force) and $6.3 \mathrm{~mm}$ for $R_{1}=16 \mathrm{~mm}$ for $\varphi=\pi / 4$. The experimental values are $x_{\dagger}=5.5 \mathrm{~mm}$ for $R_{1}=12 \mathrm{~mm}$ (the magnet hits the pipe) and $7 \mathrm{~mm}$ for $R_{1}=16$ $\mathrm{mm}$.

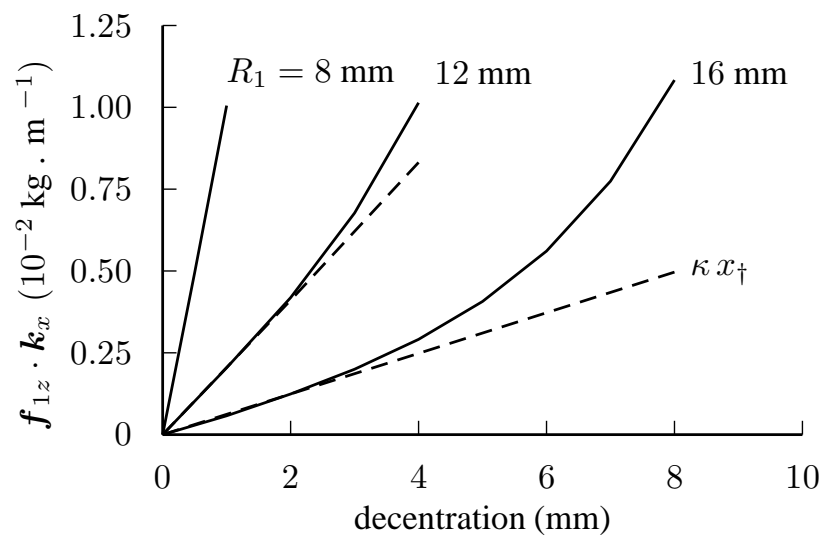

Figure 6.

Restoring force coefficients $\boldsymbol{f}_{1 z} \cdot \boldsymbol{k}_{x}$ as a function of $x_{\dagger}$ $(e=1 \mathrm{~mm})$

\section{Conclusion}

The force exerted on a magnet which falls in a conducting pipe, with a fixed direction of magnetization, depends on this direction of magnetization and has two components. The first component is directed along the symmetry axis of the pipe and tends to brake the fall. The lowest speed is found when the direction of magnetization is parallel to the symmetry axis and the highest when it is perpendicular: the relative difference between them is about one third.

The second component, labelled lateral, is perpendicular to the axis of symmetry, and tend to decentrate the magnet when the direction of magnetization is neither parallel nor perpendicular to the axis of symmetry, even if the term of decentration in the lateral force is less than $10 \%$ of the brake force. The decentration increases with the increase of the radius of the pipe with respect to the one of the magnet.

The chosen model of computation has allowed the quantification of these force components and their parametric analysis as a function of the speeds. It has been verified by experiment as far as possible.

The validation of this model when the direction of magnetization is fixed will allow to study the full motion of the magnet, i.e. its fall with decentration and rotation, with the same methodology. The current density and magnetic fields will be found iteratively, as a function of the speeds (rotating speeds included), and the analysis of the forces and torques with respect to the decentration and direction of magnetization will allow to find the ordinary differential set of equations that the motion of the magnet verifies. 


\section{References}

Jackson J.D. (1999), “Classical Electrodynamics”, John Wiley, 880 pp.

Davis L.C. (1972), "Drag force on a magnet moving near a thin conductor", J. of Appl. Phys., vol. 43, no. 10, pp. 4256-4257.

Iniguez J., Raposo V., Hernandez-Lopez A., Flores A.G., and Zazo M. (2004), "Study of the conductivity of a metallic tube by analysing the damped fall of a magnet", Eur. J. Phys., vol. 25, pp 593-604.

Levin Y., da Silveira F.L. and Rizzato F.B. (2006), "Electromagnetic braking: A simple quantitative model” Am. J. Phys., vol. 74, no. 9, pp. 815-817.

Partovi M.H. and Morris E.J. (2006), "Electrodynamics of a magnet moving through a conducting pipe", Can. J. Phys., vol. 84, pp. 253-271.

Roy M.K., Harbola M.K., and Verma H.C. (2007), "Demonstration of Lenz's law: Analysis of a magnet falling through a conducting tube", Am. J. Phys., vol. 75, no. 8, pp. 728-730.

Saslow W.M. (1992), "Maxwell's theory of eddy currents in thin conducting sheets and applications to electromagnetic shielding and MAGLEV", Am. J. Phys., vol. 60, pp. 693-711.

Bae J.S., Hwang J.H., Park J.S. and Kwag D.G. (2009), "Modeling and experiments on eddy current damping caused by a permanent magnet in a conductive tube", J. of Mechanical Science and Technology, vol. 23, no.11, pp. 3024-3035.

Nestleroth J.B. and Davis J.R. (2007), "Application of eddy currents induced by permanent magnets for pipeline inspection", NDT \& E International, vol. 40, no. 1, pp. 77-84.

Donoso G., Ladera C.L. and Martin P. (2009), "Magnet fall inside a conductive pipe: motion and the role of the pipe wall thickness" Eur. J. of Physics, vol. 30, no. 4, pp. 855-869.

Conway J.T. (2008), "Noncoaxial Inductance Calculations Without the Vector Potential for Axisymmetric Coils and Planar Coils", IEEE Trans. Mag., vol. 44, no. 4, pp. 453-462.

Levin Y. and Rizzato F.P. (2006), "Superconducting pipes and levitating magnets", Physical Review E, vol. 74 (2), no. 6, pp. 066605.1-066605.7.

Donoso G., Ladera C.L. and Martin P. (2011), "Damped fall of magnets inside a conducting pipe" Am. J. Phys., vol. 79, no. 2, pp. 193-200.

Dular P., Remacle J.-F., Henrotte F. et al. (1997), "Magnetostatic and magnetodynamic mixed formulations compared with conventional formulations", IEEE Trans. on Magn., vol. 33, no. 2, pp. 1302-1305.

Dufour S. and Vinsard G. (2007), "Iterative computation of induced currents in a three-dimensional conducting domain”, Eur. Phys. J. Appl. Phys, vol. 39, no. 2,pp. 175-178.

Strain J. (1995), “Locally corrected multidimensional quadrature rules for singular functions",SIAM Journal on Scientific Computing archive, vol. 16 , no. 4, pp. 9921017. 
Rikabi J., Bryant C.F. and Freeman E.M. (1990), "On the solvability of magnetostatic vector potential formulations", IEEE Trans. On Magn., vol 26, no. 5, pp. 2866-2874. 